\title{
Characterization of Strain-induced Defects Density in Flexible Organic Photovoltaic Cells using Capacitance Spectroscopy
}

\author{
Majid Salari $^{1^{*}}$, Ali Naseri ${ }^{2}$, and Reza Tabatabai Manesh ${ }^{3}$ \\ ${ }^{1}$ Department of Electrical Engineering, Ferdowsi University of Mashhad, \\ 9177948974, Mashhad, Iran \\ ${ }^{2}$ Department of ICT, Imam Hossein University of Tehran, 1698715461, Tehran, Iran \\ ${ }^{3}$ Researcher of the Knowledge-Based Society, Tehran, Iran \\ *salari.majid@mail.um.ac.ir
}

\begin{abstract}
The defect states have a prominent role in electrical performance of organic materials including polymers. Stretching flexible organic solar cells introduces new defects that reduce their efficiency notably. We have studied the changes of density and distribution of these defects versus strain in a roll-to-roll printed organic solar cell with P3HT:PCBM active layer. The junction capacitance spectroscopy, including $C(V)$ and $C(f)$ measurements, was employed as a novel and convenient method in solar cells under strain to characterize the density of states distribution over the band gap. $C(V)$ measurements yielded flat-band potential and the total shallow defects density and $C(f)$ spectroscopy resulted in distribution profiles. In both measurements, the junction capacitance increased with strain due to additional states contribution. The results show spreading of the distribution profile over more energy levels and more than $30 \%$ increase in total traps density for $50 \mathrm{~m} \varepsilon$ mechanical strain.
\end{abstract}

Keywords: Organic solar cells, Flexible OPV, Capacitance spectroscopy, Mechanical strain, Defect density of states

\section{Introduction}

Flexible organic photovoltaics (OPV) have received considerable attention during the past decades. Applications such as wearable and portable electronics require an adequate amount of flexibility which is one the most significant features of organic electronic devices [1-3]. This attribute, as well as other advantages, including low cost, easy fabrication and light weight, is elevating the organic materials into promising alternatives to silicon. Thus, many researchers have shown growing interest in studying the flexible organic electronics and characterizing their electrical parameters which is the first step in improving their efficiency [4-9]. Strain, bending and deformation can have negative effect on the electrical behavior of electronic devices but it is necessary for a flexible device to maintain a sufficing efficiency after such physical alterations. Previous researchers have studied the electrical behavior of organic FETs, LEDs and solar cells under strain, bending or torsion [10-12]. On one hand, experiments done on OPVs have proven that strain can change the arrangement of polymer chains and reorder the backbones along the direction of strain. As a result of this alignment, charge carriers have a more direct route from the active layer to the electrodes and this leads to higher conductivity and performance for the cell [13-15]. On the other hand, stretching a solar cell breaks interlayer connections and introduces defect states in the structural disorders formed in the layers. These defects reduce the cell's efficiency by trapping the carriers travelling through the active layer $[11,15]$. Presence of these traps was shown previously in the literature but their density has never been calculated. So detailed analysis on the density of the created trap states over energy is needed to better understand the effect of strain on 
the electrical behavior of OPVs.

Organic semiconductors have a large trap density of states (DOS) by nature and this influences their conductivity and lifetime substantially $[16,17]$. Many researchers have studied and calculated the DOS in organic materials and devices using various methods such as Deep Level Transient Spectroscopy (DLTS) [18] and Thermally Stimulated Current (TSC) [19]. In addition, capacitance characterization has been popularly employed to measure not only the DOS $[20,21]$ but also the doping density [22-24], built-in potential $[21,24]$, carrier mobility [25] and even thermal stability [26] in different materials. Capacitance spectroscopy involves measurement of the depletion capacitance $C_{j}$ over a range of biases and frequencies and finding the DOS by analyzing the capacitance-voltage, $C(V)$, and capacitancefrequency, $C(f)$, plots. The technique of using the voltage and frequency dependence of $C_{j}$ to characterize the DOS was first proposed by Hegedus et al. [27] and Walter et al. [28] in p-i-n and pn junctions. Measuring the voltage-dependent capacitance in Schottky or pn junctions leads to the traditional Mott-Schottky relation

$$
\frac{1}{C_{j}^{2}}=\frac{2}{A^{2} q \varepsilon_{0} \varepsilon N}\left(V_{b i}-V_{D C}\right)
$$

where $A$ is the junction area, $q$ the elementary charge, $\varepsilon_{0}$ the vacuum permittivity, $\varepsilon$ the relative dielectric constant of the semiconductor, $N$ the doping density, $V_{\mathrm{bi}}$ the built-in potential and $V_{\mathrm{DC}}$ the external DC voltage. As can be seen in Eq. (1), $C_{j}^{-2}$ has a linear dependence to $V_{D C}$ and a $C_{j}^{-2}-V$ plot is a straight line that its slope gives $N$ and its extrapolation intersects the voltage axis at $V_{\text {bi }}$. Furthermore, Walter et al. calculated the DOS distribution in energy gap using the capacitance spectrum over a range of frequencies. $C(f)$ is found by applying an $\mathrm{AC}$ voltage to the junction and altering its frequency. For each frequency only defect states can follow the ac signal that are not too deep. The energy level of these defects is lower than the demarcation energy,

$$
E_{\omega}=k_{B} T \ln \left(\frac{\omega_{0}}{\omega}\right)
$$

where $k_{B} T$ is the thermal energy, $\omega_{0}$ the attempt-toescape frequency and $\omega=2 \pi f$ the ac signal frequency. Finally, the defect states distribution is found using

$$
g_{t}\left(E_{\omega}\right)=-\frac{V_{b i} \omega}{q W k_{B} T} \frac{d C}{d \omega}
$$

where $W$ is the depletion width. They obtained the defect states distribution in thin film solar cells and found it decently accurate compared to earlier reports [28]. Thenceforth, this method has been applied to organic devices as well, including OPVs. For instance, characterization of the defect states in NPB [29], determination of the interfacial and deep defect states in organic solar cells [20,29-31] and investigation of trap states formed in different deposition rates [32] or traps resulted from degradation [21,33-35] are all conducted using capacitance spectroscopy in organic devices. It is worth noting that the techniques used for inorganic semiconductors are applicable to organic materials only when fitting models and parameters are employed due to the differences in band gaps, resistances and carrier mobilities. It is shown that improper application of the methods can lead to erroneous results [36,37].

Herein, we have investigated the effect of mechanical strain on the DOS distribution of organic solar cells using the aforementioned capacitance measurements. The shape and location of trap states distribution in the polymer band gap and their changes with mechanical strain provides novel insight about carriers' behavior when the cell is strained. Such information is of vital importance in flexible OPV fabrication in order to limit the device degradation under physical deformations and enhance the device performance.

\section{Experimental}

Flexible roll-to-roll printed solar cells with 3.5\% PCE and layer structure as depicted in Fig. 1 were tested under mechanical strain in this study $[38,39]$. We arranged a configuration similar to what previous researchers have used [40]. A tensile machine with $100 \mu \mathrm{m}$ resolution was used to stretch the solar cell along its length. The machine has two clamps which grip the solar cell on both sides. The clamps hold the cell tight enough to avoid slipping during the strain steps and are longer than the cell's width so they can provide a uniform force along it. Electrical measurements consisted of two circuits for $I-V$ and $C-V$ plotting. A Keithley 2400 source meter was used to obtain the $I-V$ curves accurately.

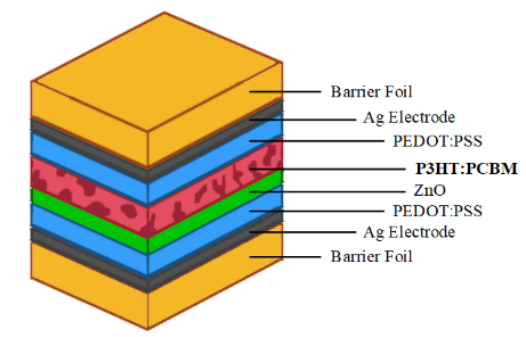

Fig. 1. Layer structure of the solar cell under study. 
Capacitance measurements were done using the circuit Kuhn employed to calculate the capacitance of MOS devices [41] (Fig. 2). This design has been used for solar cells as well because of its convenience and accuracy $[22,42]$. By applying a varying voltage with a proper frequency at the input of the circuit, we can measure the capacitance using $V_{O}=R \times I_{\text {in }}=R \times\left(V_{\text {in }} / Z_{S C}\right)$ where $Z_{S C}$ is the impedance of the solar cell. The equivalent circuit of a solar cell is shown in Fig. 3a. In this model, $I_{L}$ is the photogenerated current, $I_{D}$ is the equivalent diode current, $C_{j}$ and $C_{d}$ represent junction and diffusion capacitances and $R_{S}$ and $R_{P}$ are series and parallel resistances respectively. In the case of our study, capacitance measurements were done in dark condition, so $I_{L}=0 . I_{D}$ and $C_{d}$ are also negligible under reverse and low forward biases. Therefore, the circuit in Fig. 3a can be reduced to the elements shown in Fig. $3 \mathrm{~b}$. Since there is an insignificant voltage drop across $R_{S}$, Recart et al. ignored it in silicon solar cells [22]. However, for OPVs that are well-known for having large series resistances, this assumption is not simply true under all circumstances. The circuit of Fig. $3 \mathrm{~b}$ can be divided into two series and parallel models, red box and green circle respectively. Carr and Chaudhary tested both these circuits for a P3HT:PCBM bulk heterojunction (BHJ) solar cell and suggested a hybrid model for OPVs. They showed that, for low frequencies (below $100 \mathrm{~Hz}$ ), the capacitive impedance is extremely higher than $R_{S}$ and the parallel model can accurately be used as the equivalent circuit; but $R_{S}$ becomes comparable at frequencies higher than $1 \mathrm{MHz}$ and the series model better represents the OPV. For moderate frequencies $(100 \mathrm{~Hz}<f<1 \mathrm{MHz})$, capacitance derived from both models have the same value and either is acceptable [36]. Thus, the proper model for our frequencies of interest (lower than $10 \mathrm{kHz}$ ) is the parallel model and we can also ignore the $R_{S}$.

In order to investigate the effects of tensile strain on the DOS distribution of OPVs, we stretched the solar cell in 20 steps until the barrier foil tore and extensive cracks appeared on the active area of the

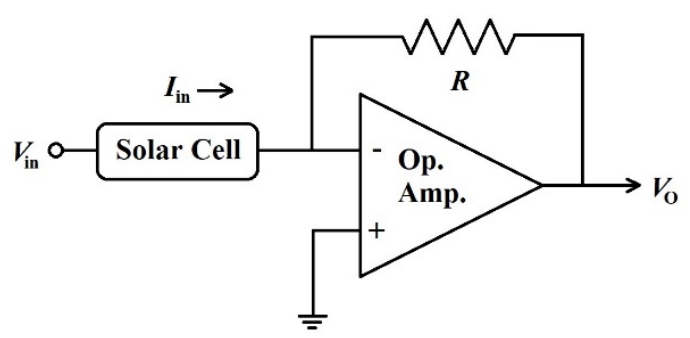

Fig. 2. Diagram of the circuit used in this project for low frequency capacitance measurements of the solar cell. cell. At each step, we increased the strain by 2500 $\mu \varepsilon$ and conducted the capacitance measurements 3 times during a 30 -minute period to make sure that the cell has reached a steady state. It is also noteworthy that all measurements are done in dark condition and the ambient temperature was monitored and fixed at $26^{\circ} \mathrm{C}$, so no additional traps are activated by light or thermal energies. The results of our experiments including $C(V)$ and $C(f)$ plots, the DOS distributions and discussions are presented in the following section.

\section{Results and discussion}

\subsection{Capacitance-voltage}

The data obtained from capacitance versus voltage measurements at $f=100 \mathrm{~Hz}$ are depicted in Fig. 4 as $C^{-2}-V$ plots. To avoid cluttering, we have only plotted curves corresponding to 5 distinctive strain steps. As can be seen, the capacitance increases ( $C^{-2}$ decreases) with strain which means higher density of charges contributing in the junction capacitance due to created defect states. Regarding low forward biases, all of the curves have almost linear behavior with slightly different slopes and an approximate intersection with voltage axis at $0.6 \mathrm{~V}$. The doping density is extracted from the slopes using Eq. (1) and displayed versus strain level in Fig. 5. The states that are too deep to follow the modulation are not activated and do not contribute in the junction capacitance and the values calculated at this frequency show the density of states that are shallow enough to follow the ac signal. This plot shows that $N$ grows gradually for up to

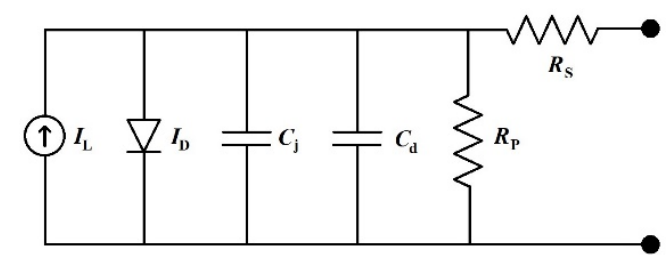

(a)

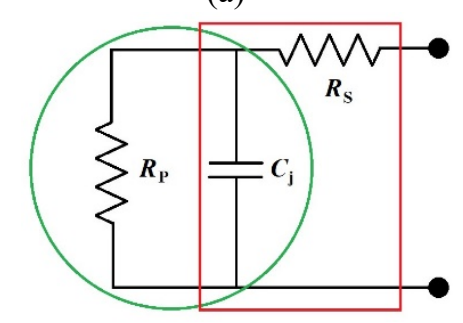

(b)

Fig. 3. (a) Electrical equivalent of a solar cell consisted of the photogenerated current $\left(I_{L}\right)$, equivalent diode current $\left(I_{D}\right)$, junction capacitance $\left(C_{j}\right)$, diffusion capacitance $\left(C_{d}\right)$, parallel resistance $\left(R_{P}\right)$ and series resistance $\left(R_{S}\right)$. (b) The simplified model of the cell under reverse and low forward biases. 


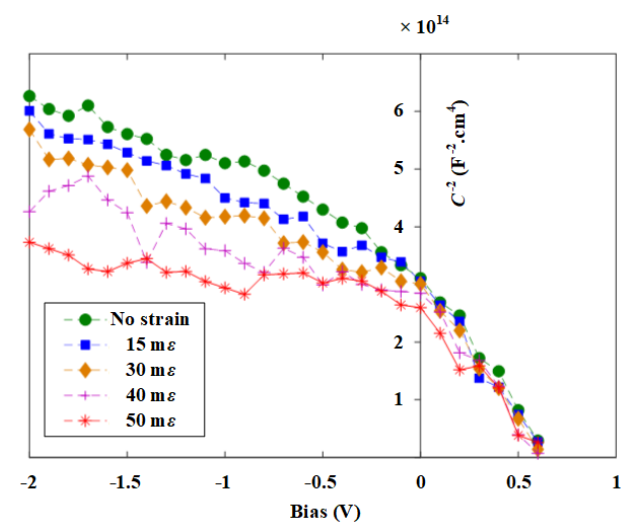

Fig. 4. $C^{-2}-V$ plots of the solar cell for 5 levels of strain at $f=100 \mathrm{~Hz}$. The curves nearly follow the Mott-Schottky relation under low forward voltages. The junction capacitance increases with strain.

$37.5 \mathrm{~m} \varepsilon$ strain and then has a sudden rise for severe strain levels. It is noted that the total growth of $N$ for $50 \mathrm{~m} \varepsilon$ is less than $10 \%$ of the initial density.

\subsection{Capacitance-frequency}

We also measured the junction capacitance versus frequency so as to achieve the DOS distribution over the polymer band gap. Figure 6 illustrates the $C(f)$ plots at zero bias for the same 5 strain steps mentioned in the last section which all experienced consistent decline with increasing frequency. Moreover, it shows that the capacitance rises as the cell is stretched meaning a reduction of the capacitive impedance, $Z_{C}$, versus strain. Since it is known that $R_{S}$ increases with strain level as well [40], it may question the validity of the parallel model of Fig. $3 \mathrm{~b}$ for the utmost tensions. So we extracted $R_{S}$ values from the $I-V$ curves using the method proposed by Werner [43] which has been used in previous studies as well $[15,40]$. As it is depicted in Fig. 7, $R_{S}$ increment for the highest strain level is less than $25 \%$ of its initial value. In addition, the inset shows the ratio of the parallel model impedance, $Z_{C \| R p}$, to $R_{S}$ at $f=10 \mathrm{kHz}$. According to this plot, for the extreme case of measurement at the highest frequency and strain level, the ratio is still

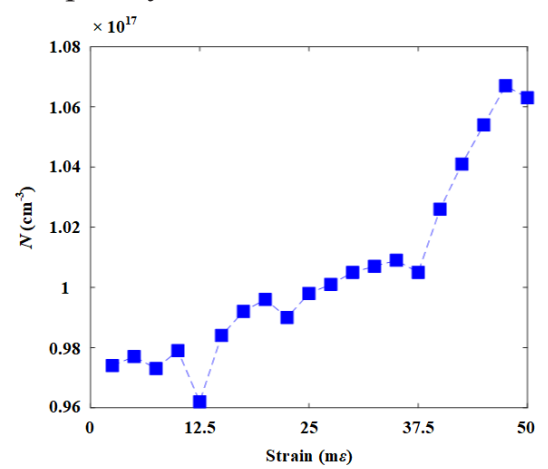

Fig. 5. Doping density versus tensile strain extracted from $C-V$ plots using Mott-Schottky relation.

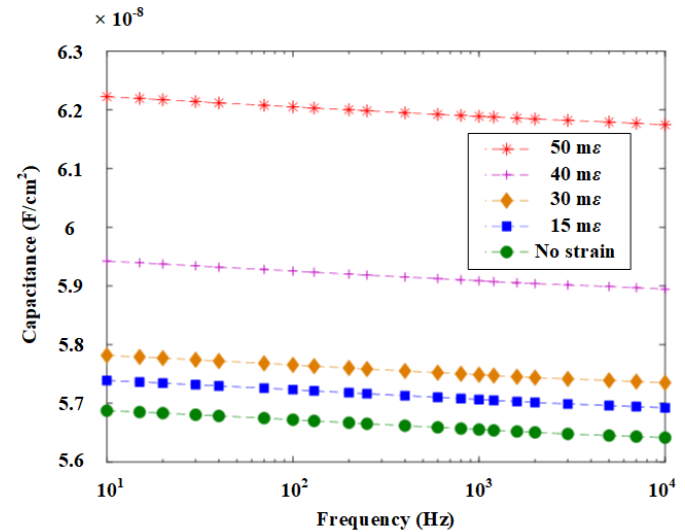

Fig. 6. $C-f$ plots of the solar cell for 5 levels of strain at zero bias. Capacitance increases with strain and decreases with frequency.

larger than 40. This confirms the accuracy of the parallel model for the whole experiment because $R_{S}$ is never comparable to $Z_{C \mid R p}$.

Concerning the DOS calculation using $C-f$ measurements, it should be noted that this technique yields the densities over a range of energies achieved from Eq. (2). Therefore, this energy range is limited by the measurement frequency sweep and in our study, it comprises energy levels from 239 to $419 \mathrm{meV}$ above the Highest Occupied Molecular Orbital (HOMO) of P3HT. It is noteworthy that another important characteristic of the organic semiconductors is the attempt-to-scape frequency which has a substantial role in the DOS characterizations [44]. Here, we have used $f_{0}=10^{8}$ $\mathrm{Hz}$ that was computed by Carr et al. particularly for P3HT:PCBM blends and is evidently much smaller than the typical value used for Silicon $\left(10^{12} \mathrm{~Hz}\right)$ [37]. As mentioned in the previous section, $V_{\text {bi }}$ values with insignificant dependence to strain were extracted from $C(V)$ curves using Eq. (1). The DOS

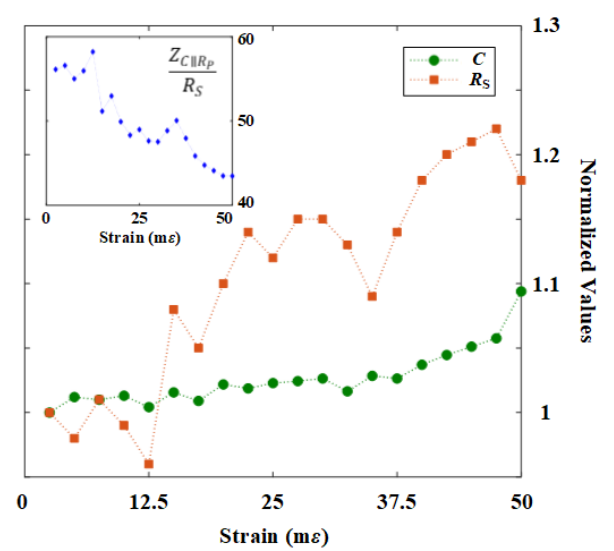

Fig. 7. The junction capacitance and series resistance of the solar cell versus strain. The inset displays the ratio of the parallel model impedance to series resistance versus strain at the highest measurement frequency, $10 \mathrm{kHz}$. For the extreme case of measurement, this ratio is still larger than 40 . 


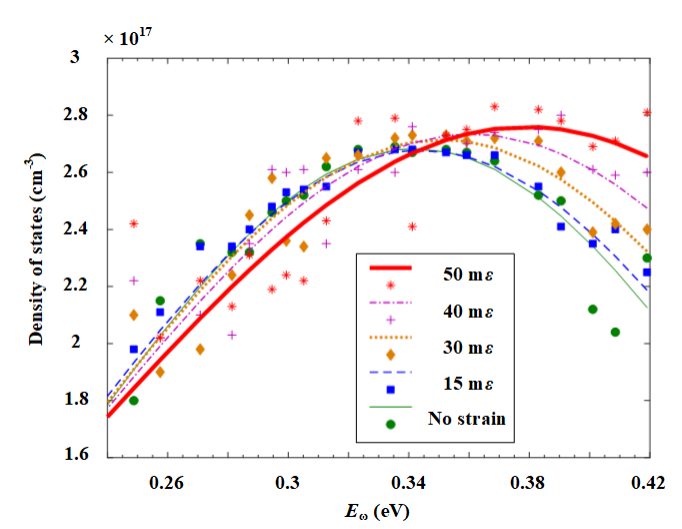

Fig. 8. DOS distribution profiles over energy for 5 strain levels. The symbols are experimental data extracted from $C-f$ plots and lines are the best fitted Gaussian distributions.

distribution obtained by applying Eq. (3) to the $C(f)$ data is demonstrated in Fig. 8. The scattered symbols are experimental data calculated from the measurements while the lines are the best fitted Gaussian distributions plotted using

$$
g_{t}\left(E_{\omega}\right)=\frac{N_{t}}{\sqrt{2 \pi} \sigma_{t}} \exp \left[-\frac{\left(E_{0}-E_{\omega}\right)^{2}}{2 \sigma_{t}^{2}}\right]
$$

where $N_{t}$ is the total density of traps, $\sigma_{t}$ is the disorder width of the distribution and $E_{0}$ denotes the energy level at the maximum of the DOS profile. Despite noisy experimental data especially in high and low energy levels, they are still distributed with a nearly Gaussian shape. Figure 9 displays the trend of the distribution parameters versus strain to better understand the effect of mechanical strain on the DOS distribution. The curves in this figure present the parameters normalized to their starting values in no strain condition. Although all 3 curves have an upward trend through the entire stretching process, changes can be divided into two main parts; for mild and moderate strain levels $(<40 \mathrm{~m} \varepsilon)$, the parameters grow with a gentle slope while higher tension results in an abrupt rise in the figures during the last steps. Through the entire process of stretching from the relaxed state to maximum strain of $50 \mathrm{~m} \varepsilon$, tensile strain has formed new deep trap states inside the band gap resulting in an increase of the total defects density from $7.6 \times 10^{16} \mathrm{~cm}^{-3}$ to $1 \times 10^{17} \mathrm{~cm}^{-3}$ along with $28 \%$ rise of $\sigma_{t}$ appeared as broader distribution profiles; regarding $E_{0}$, the profiles also move by the total amount of $37 \mathrm{meV}$ toward deeper states as the cell is stretched. These newly formed defects are the result of structural disorders and layers detachment during the strain process which reduce the polymer's conductivity and the device performance by capturing the charge carriers. Further study can be done using the techniques such as point-to-point

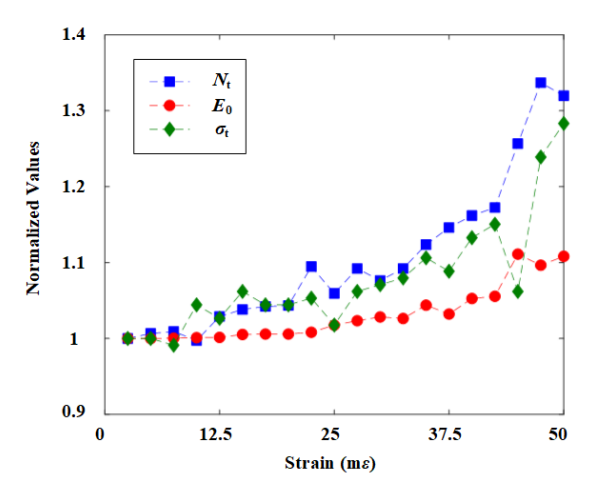

Fig. 9. Changes of the distribution parameters with increasing strain. The parameters are normalized to their initial values.

$C-V$ and drive level capacitance profiling to obtain the spatial distribution of trap states [45]. The density of defect states at layer interfaces, in the bulk and near cracks will reveal the major trap locations developed in the strained OPV.

\section{Conclusion}

We have calculated the density of trap states in a strained flexible organic solar cell using capacitance measurements. The capacitance characterization included measuring voltage-dependent and frequency-dependent capacitance and resulted in the total trap density and the DOS distribution. Using this accurate method for an OPV under tensile strain provided us with the changes of the defect densities with mechanical strain. The results showed an increase in the total density and the width of the density distribution over the band gap of the polymer versus strain. The defects are created in strain-induced structural disorders and act as traps for charge carriers inside the active layer. The outcome of this study is of most benefit to researchers for better understanding the electrical behavior of flexible OPVs under strain and to industries for improving the performance of flexible devices.

\section{References}

1. S. Berny, N. Blouin, A. Distler, H. J. Egelhaaf, M. Krompiec, A. Lohr, O. R. Lozman, G. E. Morse, L. Nanson, A. Pron, T. Sauermann, N. Seidler, S. Tierney, P. Tiwana, M. Wagner, and H. Wilson, Adv. Sci., 3 (2015) 1500342.

2. T. F. O'Connor, A. V. Zaretski, S. Savagatrup, A. D. Printz, C. D. Wilkes, M. I. Diaz, E. J. Sawyer, and D. J. Lipomi, Sol. Energy Mater. Sol. Cells, 144 (2016) 438.

3. P. C. Y. Chow and T. Someya, Adv. Mater., 32 (2020) 1902045.

4. S. Sharma, K. K. Jain, and A. Sharma, Mater. 
Sci. Appl., 6 (2015) 1145.

5. M. A. Green, Prog. Photovoltaics Res. Appl., 9 (2001) 123.

6. J. Yan and B. R. Saunders, RSC Adv., 4 (2014) 43286.

7. R. Zohourian Aboutorabi and M. Joodaki, Org. Electron., 25 (2015) 184.

8. H. Matsutani, N. Arima, Y. Ishikawa, M. Matsunaga, and Y. Okada, J. Photopolym. Sci. Technol., 32 (2019) 501.

9. T. Miyasaka and M. Ikegami, J. Photopolym. Sci. Technol., 23 (2010) 269.

10. H. Ling, S. Liu, Z. Zheng, and F. Yan, Small Methods, 2 (2018) 1800070.

11. M. Finn, C. J. Martens, A. V. Zaretski, B. Roth, R. R. Søndergaard, F. C. Krebs, and D. J. Lipomi, Sol. Energy Mater. Sol. Cells, 174 (2018) 7.

12. M. Joodaki and M. Salari, Org. Electron., 59 (2018) 230.

13. M. Bernardi, M. Giulianini, and J. C. Grossman, ACS Nano, 4 (2010) 6599.

14. B. O'Connor, R. J. Kline, B. R. Conrad, L. J. Richter, D. Gundlach, M. F. Toney, and D. M. DeLongchamp, Adv. Funct. Mater, 21 (2011) 3697.

15. M. Salari, M. Joodaki, and S. Mehregan, Org. Electron., 54 (2018) 192.

16. M. M. Mandoc, B. de Boer, G. Paasch, and P. W. M. Blom, Phys. Rev. B, 75 (2007) 193202.

17. H. F. Haneef, A. M. Zeidell, and O. D. Jurchescu, J. Mater. Chem. C, 8 (2020) 759.

18. D. A. Batovski and C. M. Hardalov, J. Appl. Phys., 74 (1993) 291.

19. A. Hepp, N. Von Malm, R. Schmechel, and H. Von Seggern, Synth. Met., 138 (2003) 201.

20. L. N. S. Murthy, D. Barrera, L. Xu, A. Gadh, F. Y. Cao, C. C. Tseng, Y. J. Cheng, and J. W. P. Hsu, J. Phys. Chem. C, 123 (2019) 10795.

21. Anjusree S., Arya K. R., and B. C. Das, RSC $A d v ., 10$ (2020) 24882.

22. F. Recart and A. Cuevas, IEEE Trans. Electron Devices, 53 (2006) 442.

23. G. D. Sharma, D. Saxena, and M. S. Roy, Synth. Met., 123 (2001) 189.

24. A. J. Campbell, D. D. C. Bradley, E. Werner, and W. Brütting, Synth. Met., 111 (2000) 273.

25. J. V. Li, A. M. Nardes, Z. Liang, S. E. Shaheen, B. A. Gregg, and D. H. Levi, Org. Electron., 12 (2011) 1879.

26. M. Tessarolo, A. Guerrero, D. Gedefaw, M. Bolognesi, M. Prosa, X. Xu, M. Mansour, E. Wang, M. Seri, M. R. Andersson, M. Muccini, and G. Garcia-Belmonte, Sol. Energy Mater. Sol. Cells, 141 (2015) 240.

27. S. S. Hegedus and E. A. Fagen, J. Appl. Phys. 71 (1992) 5941.

28. T. Walter, R. Herberholz, C. Müller and H. W. Schock, J. Appl. Phys., 80 (1996) 4411.

29. S. Kumar, U. K. Verma, and Y. N. Mohapatra, Springer Proc. Phys., Springer Science And Business Media, LLC, (2019) 1065.

30. J. Zhang, M. H. Futscher, V. Lami, F. U. Kosasih, C. Cho, Q. Gu, A. Sadhanala, A. J. Pearson, B. Kan, G. Divitini, X. Wan, D. Credgington, N. C. Greenham, Y. Chen, C. Ducati, B. Ehrler, Y. Vaynzof, R. H. Friend, and A. A. Bakulin, Adv. Energy Mater., 9 (2019) 1902145.

31. V. V. Brus, A. K. K. Kyaw, P. D. Maryanchuk, and J. Zhang, Prog. Photovoltaics Res. Appl., 23 (2015) 1526.

32. A. Sharma, S. Yadav, P. Kumar, S. Ray Chaudhuri, and S. Ghosh, Appl. Phys. Lett., 102 (2013) 143301.

33. B. Ecker, J. C. Nolasco, J. Pallarés, L. F. Marsal, J. Posdorfer, J. Parisi, and E. Von Hauff, $A d v$. Funct. Mater, 21 (2011) 2705.

34. R. A. Street, Y. Yang, B. C. Thompson, and I. McCulloch, J. Phys. Chem. C, 120 (2016) 22169.

35. F. Fungura, W. R. Lindemann, J. Shinar, and R. Shinar, Adv. Energy Mater., 7 (2017) 1601420.

36. J. A. Carr and S. Chaudhary, Appl. Phys. Lett., 100 (2012) 213902.

37. J. A. Carr, M. Elshobaki, and S. Chaudhary, Appl. Phys. Lett., 107 (2015) 203302.

38. F. C. Krebs, M. Hösel, M. Corazza, B. Roth, M. V. Madsen, S. A. Gevorgyan, R. R. Søndergaard, D. Karg, and M. Jørgensen, Energy Technol., 1 (2013) 378 .

39. M. Hösel, R. R. Søndergaard, M. Jørgensen, and F. C. Krebs, Energy Technol., 1 (2013) 102.

40. M. Salari and M. Joodaki, IEEE Trans. Device Mater. Reliab., 19 (2019) 718.

41. M. Kuhn, Solid State Electron., 13 (1970) 873.

42. Y. H. Shing and R. O. Loutfy, J. Appl. Phys., 52 (1981) 6961.

43. J. H. Werner, Appl. Phys. A Solids Surfaces, 47 (1988) 291

44. P. P. Boix, J. Ajuria, I. Etxebarria, R. Pacios, and G. Garcia-Belmonte, Thin Solid Films, 520 (2012) 2265.

45. H. S. Pang, H. Xu, C. Tang, L. K. Meng, Y. Ding, J. Xiao, R. L. Liu, Z. Q. Pang, and W. Huang, Org. Electron., 65 (2019) 275. 